\title{
Le portrait de la femme médiévale des pays d'oc: entre fiction et réalité
}

Marie Rose Bonnet

Université de Provence

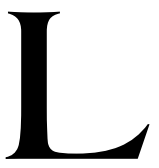

a description de la donna des troubadours appartient aux topiques de la lyrique troubadouresque: elle est jeune, belle, blanche, blonde, lumineuse, accueillante, avenante, instruite, aimable, rieuse, gaie. A ces qualificatifs, il faut ajouter la santé, certains y renvoyant implicitement. Ce portrait type est en fait celui d'une femme quasi désincarnée. Parfois cependant, une allusion est faite aux soins, à l'hygiène, aux vêtements, et une esquisse de portrait plus pittoresque apparait. Il peut donc être intéressant de partir à la recherche de ces indices et de les comparer à ce que révèlent les textes non littéraires, même si plusieurs époques sont alors concernées. La première chose que le troubadour voit, c'est le vêtement. Mais le portrait va se préciser, et le corps de la dame se dessine quelque peu. Enfin, tout cela est dû aux soins qu'elle prend, à une hygiène de vie, qui transparaît aussi à travers les textes littéraires. ${ }^{1}$

Si la littérature offre peu de portraits, les textes non littéraires n'en offrent aucun, car leur réalité ne propose que peu d'intérêt. Seul le portrait orienté, inspirateur de sentiments, intéresse alors, et c'est l'iconographie qui, en fait, renseigne le mieux. Cependant, quelques notations, glissées de temps en temps, donnent à voir certaines parties du corps féminin. C'est ainsi que dans Jaufre, ${ }^{2}$ par exemple, l'auteur écrit:

\footnotetext{
${ }^{1}$ Cet article est le complément du "Nu chez quelques troubadours", paru dans Le Nu et le Vêtu au Moyen Age (XIIe-XIIIe siècles) Sénéfiance no 47, P.U.P., 2001. ${ }^{2}$ Les troubadours. Jaufre. Flamenca. Barlaam et Josaphat. Traduction de René Lavaud et René Nelli. Bibliothèque Européenne, 1960. p. 161.
} 
Una piusela, qe nunc cre

Qe al mun n'aja belasor

Car pus ac fresca la color

Qe rosa, cant es ades nada

E fu sa gonela esquintada

Tro aval desos la tetina

Qe ac plus blanca qe farina...

(Le plaisir qu'elle prend à se parer est cependant considéré, dans la réalité, comme une manifestation de son orgueil et de son désir de perdre les hommes, alors que la littérature troubadouresque en fait un motif narratif d'attraction.)

Le corps nu de la demoiselle, ou plutôt sa poitrine, se révèle dans la citation précédente, faisant ressortir sa blancheur. Le portrait de Brunissens, toujours dans Jaufre, est lui aussi valorisant. ${ }^{3}$ Après avoir vanté sa beauté, "sos oils e sa bela cara", le poète en donne la vision suivante:

Car pus es fresca, bela e blanca

Qe neus gelada sutz en branca

Ni qe rosa ab flor de lis.

Qe sul ren non a mas asis,

Decovinen ni laig estan.

Aixi es faita per garan,

Qe non $i$ a ops mais ni meins.

E sa boca es tan plasens

Qe par, qi ben la vol garar,

C'ades diga c'om l'an baisar.

Plus tard, lorsque Jaufre la rencontre, il ne peut s'empêcher d'admirer:

Sun fron e sun col e sa cara,

Qe fo fresca e blanca e clara,

Sa boca e sus oils plasens,

Clars e amoros e risens, ${ }^{4}$

Les vers 7137 à 7151 continuent ce portrait:

E fon vestida coindament

D'un cisclaton mot autament,

${ }^{3}$ Jaufre, o.c., p. 202.

${ }^{4}$ Jaufre, o.c., p. 226. 
E siei cabel delgat et saur

Son gent estreit d'un filet d'aur;

Es a bella cara plasent

On anc non ac affaitament,

Anz es ben fina per natura,

Qu'en nulla sason non pejura,

Plus al matin que al colcar,

Mais ben la ves om mellurar;

Rellusi et geta clardat,

Que tuit en son enluminat

Cels que l'anavon environ;

E ac un capel de paon

En son cap mes per la calor,

E portet en man una flor

Mot bella e mot ben flairan.

Enfin, des vers 7700 à 7702, d'autres détails sont donnés:

E ac un prim filet de cilhas,

Negre et sotil e delgat,

Natural e ben faissonat,

Que nun fun pelat ni tundutz.

Tout cela parce que "Deutz la fet per meravilhas"... Si nous exceptons les vêtements, ce qui ressort ici est son aspect naturel. Cela correspond à une conception médiévale qui veut que Dieu a créé la beauté, et que seule la beauté naturelle est valable; les clercs notamment vont donc vilipender fréquemment les femmes qui se maquillent. Modifier leur apparence, se maquiller, sont autant de preuves qu'elles ne se satisfont pas de ce que la nature, et donc Dieu, leur ont octroyé. C'est pourquoi Brunissens, personnage positif, est présentée comme très belle, et sachant respecter la nature sans chercher à la cacher par des artifices qui terniraient cette luminosité qui émane d'elle. D'autres passages, dans d'autres oeuvres, insistent sur cette beauté naturelle. La jeune fille courtoise n'utilise pas d'artifices pour être belle, elle n'en a pas besoin. Les troubadours rejoignent ainsi la conception religieuse.

5 "Tenson entre Dieu et le moine de Montaudon", Les troubadours. Le trésor poétique de l'Occitanie. Textes et traduction par René Nelli et René Lavaud, B. E., 1966, p. 822. 
Certains critiquent de plus le maquillage, anti-naturel, comme cela apparaît dans une tenson entre Dieu et le moine de Montaudon: ${ }^{5}$

De las dompnas que-s van peignan

[...]

Qu'an faich lo teing carzir,

Que se fan la cara luzir

[...].

Dans une autre canso, nous trouvons

De las dompnas, cui natura

Es que lor caras teignan gen.

Une autre mentionne "Que dompna-s genz'ab penchura". Dans une tenso, "Quan tuit aquist clam foron fat", le moine de Montaudon, toujours lui, rapporte les plaintes des tableaux, des images, qui reprochent aux femmes de les priver de leur peinture à vouloir trop les utiliser pour elles. Il va de plus indiquer comment se préparaient les fards dans un sirventès, "Sobre sacramen vei obrar": ${ }^{6}$

Que-ill quecha fai pisar son glut

Am ueu pastat

De blanquet e de vermeillon

Se meton tant sobre-l menton

E en la fatz

[...]

De çafra e de tifeigno,

D'angelot, de borrais an pro

E d'argentat, De que se peingnon a bando

Quan l'an mesclat.

En lait de sauma an temprat

Favas, ab so que s'an adobat

Lo viel cortves

[...]

Quant ellas an lor onguimentz

Totz ajustatz per sagramentz,

Vos veiriatz

de boissas e de sacs tresentz

Ensems liatz.

${ }^{6}$ Le moine de Montaudon, o.c., p. 830. 
La critique est féroce, certes; cependant, nous pouvons supposer que l'auteur s'appuie, tout en les exagérant, sur des pratiques courantes qui contredisent le principe de beauté naturelle tant chanté par les autres troubadours. Cercamon, dans Ab lo temps qe fai refreschar, associe le maquillage au mensonge, et préfère une dame qui "non es vernisada". L'association naturel-beauté apparaît ainsi chez les troubadours, de manière positive ou négative, explicite ou implicite, et la donna, pour être aimée, ou vénérée, ne doit se présenter que sans fard.

Cela ne signifie pas qu'elle ne prenne pas soin d'elle, et qu'elle n'ait pas une bonne hygiène de vie. Il existe en fait deux catégories de soins: ceux qui permettent d'entretenir la beauté et une bonne santé, ceux qui tentent de réparer un outrage du temps signalé aussi dans certaines cansos. C'est ainsi par exemple que Bertran de Born ${ }^{7}$ la décrit lorsque le temps a fait son oeuvre:

cant ilh a la pel larja

Qe li ruon li costat e li flanc,

Et a los oils plus vermeils d'un envarc

E-l cap canut e par de col retomba.

Ces vers rappellent par ailleurs le poète Horace, quelquefois cité par les troubadours, qui demande de profiter de la vie avant que vieillesse n'arrive. Tous ces poèmes traitent finalement du même thème du temps qui passe, et du fait qu'il faut profiter de sa jeunesse avant qu'elle ne cesse. Joven est un terme récurrent dans cette lyrique, qui renvoie à une jeunesse du coeur alliant toutes les qualités de cortesia, et de mesura. Mais, au-delà de cette connotation abstraite, il y a aussi la louange de la jeunesse effective, et certains troubadours n'hésitent pas à le dire crument.

La décrépitude du corps due à l'âge n'est pas non plus sans rappeler une autre atteinte à ce même corps évoquée dans la tenso entre Alaïs, Iselda et Carenza. ${ }^{8}$ L'une des trobairitz explique à Na Carenza qu'elle voudrait bien se marier, mais

Na Carenza, penre marit m'agenza, mas far enfantz cug qu'es grans penedenza, que las tetinas pendon aval jos e.l ventrilhs es cargatz e enojos.

${ }^{7}$ Bertran de Born, o.c., p. 840.

${ }^{8}$ Meg Bogin. Les femmes troubadours. Paris, 1978. p. 168. 
Elle préfère contrevenir à l'une des fonctions explicites de la femme, qui est de procréer. Ce sont donc bien les principes de la société seigneuriale qui se mettent en place qui sont critiqués. Les revendications de beauté et de liberté du choix en amour sont aussi des protestations, mais littéraires, et émanant de voix masculines. Quel que soit le sens que l'on donne à cette tenson, il n'en est pas moins vrai que la maternité est vue comme un élément destructeur de la beauté, au même titre que l'âge pour Bertran de Born.

Cependant, la femme dispose, pour affirmer, en quelque sorte, son identité, de l'apparence, et du vêtement. Les textes en offrent plusieurs exemples. Sont ainsi cités le "mantelh", la "gonela", une "sentura", les "gans", le "muzel", le "sisclato", la "pelissa", la "camiza", les "sotlars", les "caussas", le "sobrecot", le "bliau", les verbes "vestir" et "causar" revenant aussi fréquemment. Or, une sentence arbitrale sur un différend survenu entre deux pâtissiers de 1449, éditée par Pansier" propose des "raubas, capayrons, camisas et autras vestimentas" ainsi qu'une "sentura deladita jova garnida de argent'. D'autres documents non littéraires mentionnent les mêmes vêtements.

Par contre, les textes littéraires, s'ils indiquent parfois la qualité du tissu, ou la couleur, sont plutôt pauvres quant à une description détaillée. Il peut servir de cadeau que le troubadour offre à la dame aimée lorsqu'elle accepte son amour. Mais elle devient alors vénale aux yeux de la société. De plus, bien souvent, c'est lui qui attire d'abord le regard, et la critique. C'est aussi un signe de discrimination extrêmement puissant. Il permet ainsi de reconnaître l'honnête femme de la prostituée. Cette dernière est rejetée, car vivant en marge de la règle. Elle est exclue de la société, et des statuts municipaux règlementent les lieux qu'elle doit fréquenter, ainsi que les habits qu'elle peut porter, qui la distinguent de la "femme honnête". C'est ainsi par exemple que dans les Statuts d'Arles ${ }^{10}$ se trouvent les interdits suivants:

Item tota fema publica putain cantoniera ho tenant mal vida et inhonesta demorant en cariere de las femas de ben et bonas que porte vel en la testa bo subre son col bo espallas bo plechons bo

\footnotetext{
${ }^{9} \mathrm{P}$. Pansier. Histoire de la langue provençale à Avignon du XIIe au XIXe siècle. Marseille, 1974. p. 187.

${ }^{10}$ Ms. AA 16, Archives Communales d'Arles, 1251-1385, f 185 r.v.
} 
garlandas ho anel d'or ho d'argent sie condempnada per cascuna causa en sinquanta sols de cor. et en perdement de las causas susdichas et tota fama honesta et de ben les ly puesca levar sens encorre denguna pena. Et assi los vezins et las vezinas las puescan gitar de leur carriera de leur auctoritat et sens encorre denguna pena.l Item tota fema publica ou cantoniera et inhonesta que vaga per Arle sens agulheta subre la roba sobeyrana que portarie sie condempnada en sinquanta sols cor. et en perdement de las raubas.

Cela montre une nouvelle fois que, comme dans de nombreuses villes, car Arles n'est pas une exception, la prostituée, notamment la plus aisée, veut ressembler à la bourgeoise, malgré les interdits discriminatoires, et les statuts arlésiens insistent là-dessus. De plus, si elle n'a pas le droit de se trouver dans les quartiers des notables, elle peut rester dans les plus populaires. Le vêtement a donc bien une fonction sociale; marqueur d'une classe, d'une position, il va être porteur d'un symbolisme social qui éloigne la femme, car il cache, en quelque sorte, la nature. Il participe aussi de la luxure, considérée comme un péché par les moralistes chrétiens, et l'un des moyens dont elle use volontiers pour se démarquer de la condition dans laquelle on veut l'enfermer. Or, si nous en croyons Matfre Ermengau, ${ }^{11}$

\section{E porto, per gran malvastat, Lor vestimen fort escolat, E van fort espeytrinadas, E mostro lurs carunhadas.}

Il y a ici renversement de situation, puisque le vêtement l'assimile à la prostituée. Littérature et réalité deviennent alors deux miroirs déformants qui se renvoient une image inversée. Selon J.-M. Cabasse, ${ }^{12}$

Les mères de famille ne doivent pas côtoyer les filles; elles ne doivent pas se confondre, et on ne doit pas pouvoir les confondre-d'où les règlementations vestimentaires qui se multiplient à partir du XIVe siècle à la fois en droit canonique et dans les statuts urbains. Ces dispositions d' ornatu mulierum ne sont pas seulement des mesures

\footnotetext{
${ }^{11}$ Matfre Ermengau, o.c., p. 720.

${ }^{12}$ J.-M. Cabasse. "La condition de la femme mariée en Languedoc (XIIIe-XIVe siècles)”; Cabiers de Fanjeaux no 23, p. 99-112.
} 
d'ordre économique destinées à limiter des prodigalités vestimentaires que la crise rendait particulièrement choquantes, ce sont aussi des mesures d'ordre moral destinées à lutter contre l'étalage du luxe et la contagion de la luxure; ce sont encore des mesures d'ordre social destinées à éviter la 'confusion des genres',

et les codes vestimentaires très stricts tentent de règlementer les comportements. J. Le Goff l'a bien analysé lorsqu'il écrit: "Dans la société féodale ces codes ont fonctionné avec une efficacité particulière, car ils avaient une place essentielle dans le statut social et dans le système de valeurs". ${ }^{13}$ Or, la littérature décrit le plus souvent une femme bien habillée, voire aux vêtements somptueux, correspondant au rang social qu'elle occupe dans la société hiérarchisée qui fait du vêtement un signe de reconnaissance. Ils sont indissociables de la vie de cour, de la cortesia, et de la fin'amor. L'ambivalence du portrait qui se déploie ici témoigne bien de cette ambiguité de l'image littéraire d'une donna, ou d'une jeune fille qui, pour tenir son rôle, contrevient à des principes édictés par une morale restrictive, mais se fie aussi à sa beauté naturelle pour plaire. Le vêtement révèle donc l'être, finalement, plus que le paraître. Sa fonction littéraire rejoint une revendication de liberté de la femme qui ne veut pas obéir à des règles cherchant à faire coïncider son apparence avec un statut social qui fait d'elle une mineure; dans la lyrique troubadouresque, elle est la donna, et son apparence n'est que le témoignage de cette volonté, mise en scène cependant par un discours masculin.

Les comparaisons, traditionnelles, correspondent bien, par ailleurs, à ce que chantent constamment les troubadours lorsqu'ils évoquent leur donna. Falquet de Romans, ${ }^{14}$ lorsqu'il décrit celle pour qui il chante, utilise des procédés vagues qui relèvent plutôt de l'énumération:

et a be plus fressca color

qe rosa no flors d'angilen,

bella bocha, bels uogltç e nars.

De même, dans un autre poème, le poète détaille les différentes parties du corps:

${ }^{13}$ J. Le Goff. L'imaginaire médiéval. Paris, 1985. p. 168.

${ }^{14}$ Raymond Arveiller et Gérard Gouiran. L'oeuvre poétique de Falquet de Romans troubadour, publications du C.U.E.R.M.A., 1987. p. 50. 


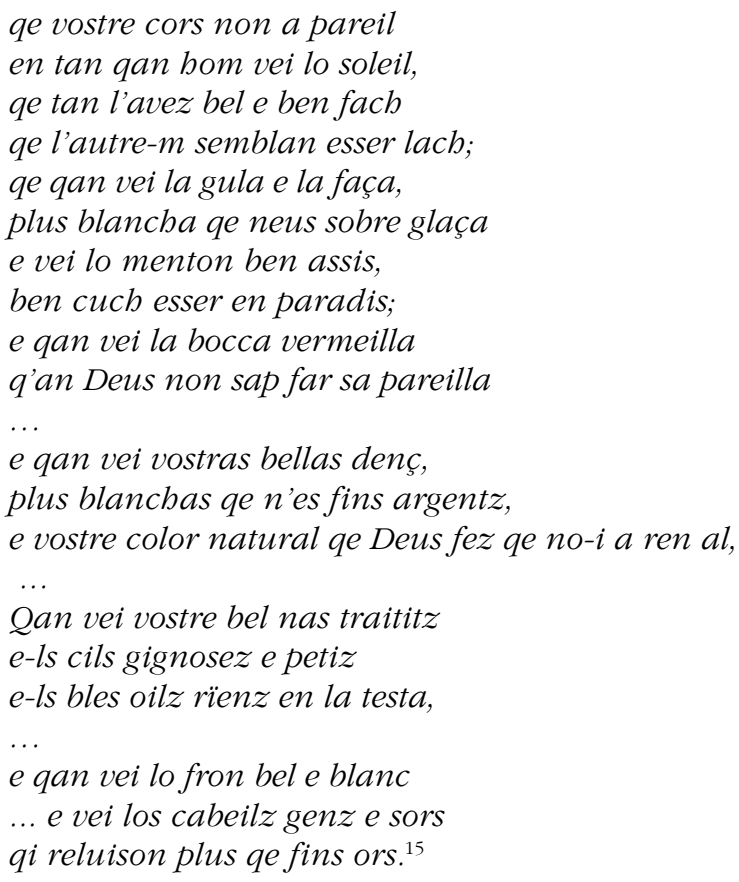

Nous retrouvons les mêmes images, les mêmes comparaisons, les mêmes couleurs aussi, ainsi que cette idée de beauté naturelle, oeuvre de Dieu. De plus, les couleurs qui ressortent sont, en dehors de l'or, le rouge et le blanc, qui ne sont pas sans évoquer Perceval le Galois perdu dans ses pensées lorsqu'il voit dans la neige les gouttes de sang de l'oie qui lui rappellent aussitôt celle qu'il aime. Le corps nu de la dame, baptisée ensuite Constance, ou suggéré par le peu de vêtements qu'elle porte, apparaît aussi, par exemple dans Le livre des aventures de monseigneur Guilhem de la Barra ${ }^{16}$ Lors du baptême de Mauléon, une dame est présente:

\footnotetext{
${ }^{15}$ R. Arveiller et G. Gouiran, o.c., p. 162-164.

${ }^{16}$ Arnaud Vidal de Castelnaudary. Le livre des aventures de monseigneur Guilhem de la Barra. Edité et traduit en français moderne par Gérard Gouiran. Paris: H. Champion, 1997, p. 110-112.
} 


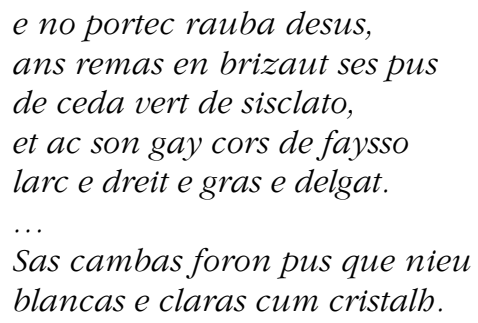

Ce type de description est utilisé aussi dans le Roman de Fierabras. ${ }^{17}$ Le portrait de Floripar, au nom déjà évocateur, relève de la même conception esthétique:

Ac lo cors bel e dreyt e ben afaysonat;

La carn aviá pus blanca qu'evori reparat,

E la cara vermelha cum roza en estat,

E la boca petita, e tenc las dens serrat,

Qu'ela avia pus blancas que neu can a gelat;

E cenh una correya de seda per baudrat;

La finela fon rica de fin aur esmerat.

La blancheur revient donc très fréquemment, et nous pourrions citer les Cansos de Guilhem de Cabestain. Dans "Ar vey qu'em vengut als jorns loncs ${ }^{18}$ par exemple, le troubadour s'exclame:
Anc pus N'Adam culbic del fust
Lo fruig don tug em en tabust
Tam bella no.n aspiret Crist:
Bel cors benestan, car e just,
Blanc e lis plus qu'us almatist

A cette blancheur se joint un autre élément, le polissage. Or, nous savons par ailleurs que les femmes, pour être le plus blanches possible, se polisaient le corps. Ce fait a suffisamment marqué Guilhem pour qu'il l'évoque à nouveau dans "Lo dous cossire":

\footnotetext{
${ }^{17}$ Les troubadours. Le trésor poétique de l'Occitanie. Texte et traduction par René Nelli et René Lavaud, B.E., p. 422.

${ }^{18}$ Les chansons de Guilhem de Cabestanh. éditées par Arthur Långfors. Paris, 1924, p. 7 et 15 .
} 


\begin{abstract}
En sovinensa
Tenc la car' e.l dous ris, Vostra valensa

E.l belh cors blanc e lis.
\end{abstract}

Parfois, des images, suggestives, appuient quelque peu la description, comme dans "Rassa, tant creis e mont'e poia" de Bertran de Born: ${ }^{19}$
Rassa, domn'ai qu'es fresqu'e fina,
Cuenda e gaia e mesquina:
Pel saur, ab color de robina,
Blanca per cors com flors d'espina,
Coude mol ab dura tetina,
E sembla conil per l'esquina.

Il est plus précis dans "Mal ofai domna cant d'amor s'atarja":
Mentr'es joves, e-l color fresc'e blanc'
E las tetinas duras ses tot embarc,
E-l ventr'es planz, senz ruas e senz comba,
E-l conz es gros e-il pelet esta-n som,
$E$ las ancas planas per meils jazer
E-l pes petitz e-l oils clars e rizens,
E-l cors fresquetz e-l pel saur en la testa.

De même, Gui d'Ussel, ${ }^{20}$ dans "En tanta guisa.men'amors", chante sa dame:

Bona dompna, pretz e valors,

E cortesi'ab gen parlar,

Huoill rizen, amoros e clar,

E gens cors ab frescas colors.

Tous ces vers offrent ainsi un portrait plus ou moins complet de la femme idéale, et idéalisée, dépeinte dans la lyrique troubadouresque.

Cependant, dans la plupart des cas, il s'agit plus d'une énumération, suivie de qualificatifs vagues ou abstraits, que d'une véritable description, même si quelques images suggestives relativisent l'impression première.

${ }^{19}$ Gérard Gouiran. L'amour et la guerre. L'oeuvre de Bertran de Born. Paris: P.U.F., 1985. p. 18; 840.

${ }^{20}$ Jean Audiau. Les poésies des quatre troubadours d'Ussel. Paris, 1927. p. 34. 
Nous pouvons alors récapituler les adjectifs qualificatifs et les termes comparés. Les éléments de portrait qui apparaissent le plus fréquemment, ainsi que les adjectifs qui les qualifient sont: la color: fresca, natural, blanca, rosana; la tetina: blanca, dura; la cara: fresca, blanca, clara, bella, plasent, fina, vermelha; la boca: plasens, bella, vermelha, petita; les oils: plasens, clars, amoros, risens, bels; le cabel, ou les pels: delgat, saur, genz; les cilhs: negre, sotil, delgat, natural, ben faissonat, gignisez, petitz; le cors: bel, ben fach, gay, larc, dreit, delgat, ben afaysonat, benestan, blanc, lis, fresquet, gens, graile; la faca: blanca; le menton: ben assis; les dens: bellas, blancas; le nas: bel; le fron: bel, blanc; les cambas: blancas, claras; la carn: blanca; le coude: mol; le ventre: planz, sens ruas, sens comba; le cons: gros; les ancas: planas; les pes: petits. Quant à la donna, elle est: fresca, bela, blanca, cuenda, gaia, mesquina. Les éléments de comparaison sont récurrents: la rosa, la rosa ab flor de lis, la rosa en estat, la flor d'angilen, la flor d'espina, la farina, la neus gelada, la neus sobre glaca, fins ors, color de robina, le lach, l'almatist, le fin argent, le cristalh, l'evori reparat, le conil.

Ce sont surtout les couleurs qui inspirent ces comparaisons, celle de la rose en particulier, symbole très répandu. Très curieusement d'ailleurs, elle peut représenter, dans l'iconographie chrétienne, une coupe, le Graal, qui recueille le sang du Christ; elle renvoie aussi à la transfiguration des gouttes de ce sang. Or, parmi les couleurs qui caractérisent le visage de la dame, le rouge et le blanc sont fréquemment associés, la pureté et le sang. De même, elle peut représenter la résurrection et l'immortalité. De plus, les troubadours lettrés ont peut-être fait le rapprochement entre rosa et ros, la pluie, la rosée, faisant alors de la rose un symbole de régénération. ${ }^{21}$ En fait, au-delà de l'utilisation traditionnelle, les comparaisons expriment aussi des symboles qui font de cette donna un être désincarné, porteur de sens.

Outre la couleur, et la dame elle-même, des parties de son corps (ce terme étant inclus) apparaissent dans ces portraits. Quelques adjectifs différents les qualifient. Ceux qui se rapportent au plus grand nombre d'éléments sont bel et blanc. Cependant, si la beauté renvoie bien à une notion esthétique, la blancheur, quant à elle, serait plutôt du domaine médical. La littérature troubadouresque, et les cansos en particulier,

${ }^{21}$ Frédéric Portal. Des couleurs symboliques, dans l'Antiquité, le Moyen-Age et les Temps Modernes. Paris, 1837. 
célèbrent la blancheur de la dame aimée. Or, d'après les traités de médecine, la blancheur serait un antidote à la mélancolie, la bile noire. La bile, noire ou jaune, agit sur le teint, et le médecin, en constatant la couleur de la peau, peut savoir s'il y a maladie ou non. La blancheur, signe de bonne santé et d'équilibre, surtout chez l'aristocrate, car la paysanne, souvent dans les champs, est brunie par le soleil, devient un signe représentatif d'une classe sociale. Et, pour cela aussi, nous savons que les femmes tentaient, par n'importe quel moyen parfois, de se blanchir la peau, en la ponçant, ou en utilisant du blanc de céruse, ce qui provoquait des cancers dont se plaignaient ensuite les médecins. Cette beauté, pour naturelle qu'elle soit, est néanmoins entretenue, car la dame doit prendre soin d'elle, ainsi que le préconise Bertran de Born "Joves se te, quan guarda son cors belh".

Parmi les éléments qui reviennent le plus, les yeux, extrêmement importants, puisque c'est bien souvent d'eux que naît l'amour. Or, la médecine médiévale leur accorde elle-aussi beaucoup d'importance, et les yeux rouge étaient souvent considérés comme malades. Mais le portrait n'est pas toujours flatteur, ainsi que nous pouvons le constater dans Jaufre: ${ }^{22}$

Una veila desotz un pi,

Qe jac estet acoutrada,

E fo pelosa e ruada,

Magra, e seca pus qe leina.

...

mas qe dreiset sun cap

Qe ac major, senes tot gap,

C'una dorca de dos cesters,

E-ls oils tan paucs can us diners,

Lagainos e esgrapelatz

E tot entorn blaus e macatz,

E las silas grans e cregudas,

E lauras grossas e morudas,

E longas e amplas las dens,

Aitan rosas can aurpimens,

Qe l'eisun deforas .iij. detz,

$E$ ac en la barba peletz,

E los grinos plus secs qe pendutz,

Las mùas pus negras qe carbo,

${ }^{22}$ Jaufre, o.c., p. 308-310. 


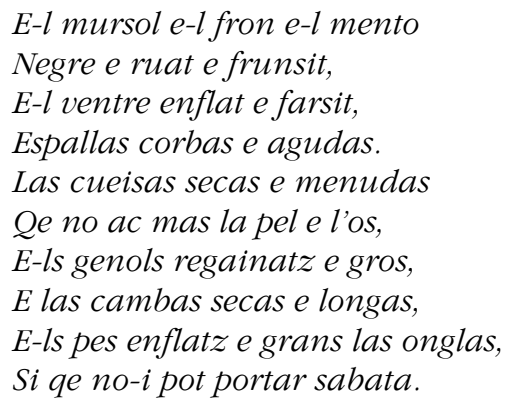

L'antithèse de la jeune femme belle, saine et naturelle, est bien la vieille femme, qui surgit au détour d'une narration qui oppose ainsi deux états, deux âges et, peut-être, l'idéal et la réalité.

Les cheveux sont quant à eux fréquemment signalés, surtout lorsqu'il s'agit de vanter leur blondeur, nous l'avons déjà vu. Les soins qui permettent de les entretenir se retrouvent dans les traités de médecine. Par exemple, au folio 33 du Tractatus medicus, le médecin Gérard de Solo explique que "mulieres Montipessulami ponunt in lexivia flores rosarum, geneste, cortice citri, radices gentiane et flores cardemoni". ${ }^{23}$ Les femmes utilisent donc des shampoings afin d'avoir des cheveux très propres. Mais ils ont d'autres fonctions narratives, et traduisent par exemple des gestes de déploration, comme dans le cas de Jaufre. ${ }^{24}$ Le roi, accompagné de Gauvain, découvre devant un moulin "Una femna qe rom sa crin". Plus tard, une autre femme intervient, "Sus cabels tiran e rumpen". ${ }^{25}$ Ensuite, Brunissens et d'autres femmes

Sun se presas al dol a far,
E viratz lurs pels arabar.

Chaque fois, cela s'accompagne de gestes des mains et d'autres manifestations. Il s'agit là d'une image ancienne, renvoyant aux gestes des pleureuses de l'Antiquité. La chevelure est aussi un motif narratif

\footnotetext{
23 "Les femmes de Montpellier mettent dans leur produit pour se laver les cheveux des fleurs de rose, du genêt, des écorces de citron, des racines de gentiane et des fleurs de cardamone".

${ }^{24}$ Jaufre, o.c., p. 50.

${ }^{25}$ Jaufre, o.c., p. 158.
} 
important dans le roman de Flamenca. ${ }^{26}$ Par exemple, le mari, jaloux, fait grief à Flamenca de ses cheveux, et la menace de les lui couper, car ils attirent, pense-t-il, les prétendants, et sont la cause de son infidélité supposée. Plusieurs vers sont ainsi consacrés à ce thème:

E vostra penchura non tolle!

...

E gens aves levat coaza,

A l'autr'an cub qu'en fares massa

Quan la-us farai ab forses tondre;

...

Dieus! qui vi mais tant bellas cris!

Plus bellals] son non es aurs fis.

En voulant lui couper les cheveux, il cherche à lui ôter sa féminité, à lui faire perdre tout signe d'indépendance, voire même d'existence. Ce motif traditionnel, signe d'infamie ou de servitude, traduit à nouveau la volonté du seigneur d'assujétir sa femme à son seul pouvoir. Mais elle prend soin de ses cheveux, et ses suivantes font de même, car c'est aussi un signe qui leur permet de ne pas montrer leur chagrin: ${ }^{27}$

Lurs crins adoban et aplanon,

Et an lurs caras ben lavadas.

Que non paresca sion ploradas.

Nous sommes bien ici dans un motif narratif qui a la même valeur que dans les exemples précédents, et qui donne à voir l'état mental dans lequel se trouve une personne, ainsi que son refus de se laisser influencer de quelque manière que ce soit. La personnalité, les conceptions transparaissent ainsi, et l'attitude face à la chevelure traduit plus l'état d'esprit dans lequel se trouve la protagoniste. L'aspect extérieur reflète donc, ou masque, l'aspect intérieur, et la femme se sert de ses cheveux comme d'un langage codé qui donne un message, celui qu'elle veut envoyer à son interlocuteur.

L'hygiène tient par conséquent une grande place dans le mode de vie féminin, et masculin. Recommandée par les traités de médecine, elle

\footnotetext{
${ }^{26}$ Flamenca, o.c., p. 702.

${ }^{27}$ Flamenca, o.c., p. 1000.
} 
permet aux femmes de plaire aux hommes. Le reproche qui leur est fait, de passer trop de temps à leurs toilettes, relève en fait de la seconde situation. Or, ainsi que le signalent G. Brunel-Lobrichon et Cl. DuhamelAmado, "Médecine et souci de coquetterie convergent vers les recettes de soins esthétiques. Certaines nourritures donnent bon teint, [...] des aromates parfument les cheveux et éloignent les poux - musc, giroffe, noix de muscade et cardamone - ou masquent l'odeur de transpiration. Pour l'haleine, outre le lavage des dents, il est conseillé de mâcher de l'anis, fenouil, cumin ou cardamone". ${ }^{28}$ De plus, médecine et nourriture complètent les préceptes esthétiques afin d'obtenir la plus belle apparence possible. L. Bolens ${ }^{29}$ rappelle par exemple que "Comme les sirops concluent également les livres de cuisine, ils rappellent que, pour le XIIIe siècle, le 'régime du corps' est un art et une science égaux en dignité avec la science des herbiers et celle de la médecine". La diététique, en liaison avec la médecine, favorise le choix de produits considérés comme bons pour la santé, et pour la beauté. Les onguents, au même titre que les confitures, participent de ce bien-être, s'ajoutant aux bains aromatiques.

Médecine et esthétique peuvent être de plus liées, et la première donne à la beauté la possibilité de perdurer. C'est ainsi que certains traités médicaux proposent des recettes et des conseils de beauté. C. Battles et $\mathrm{T}$. Vinyoles en publient par exemple un extrait. ${ }^{30}$ En outre, ainsi

${ }^{28}$ G. BRUNEL-LOBRICHON, Cl. DUHAMEL-AMADO. Au temps des troubadours B XIIe-XIIIe siècles. La vie quotidienne, 1997. p. 178.

${ }^{29}$ L. Bolens. "Les sorbets andalous (XIe-XIIe siècles) ou conjurer la nostalgie par la douceur". Du manuscrit à la table. Essais sur la cuisine au Moyen-Age et répertoire des manuscrits médiévaux contenant des recettes culinaires. P.U. de Montréal, 1992. p. 257-272.

${ }^{30}$ C. BATTLE et T. VINYOLES. "La culture des femmes en Catalogne au MoyenAge tardif”, La Femme dans l'histoire et la société méridionales, (IXe-XIXe siècle), Montpellier, 1995, p. 149: "(Biblioteca de la Universitat de Barcelona, ms. 68). E per ço, en lo present libre intitulat 'Flos de les dites receptes', lo qual libre, senyores, no us e presumit fer creer que aquel ayats al presenr nessesari per esmenar en res la beutat, gentilessa e poritat de vostres persones, com d'aquelles vos aya Déu prou bestantment dotades entre les altres dones qui de semblants gracies se poden alegrar; mas aquel vos e fet e compost per tal que per alguns achcidents qui sesdevenen disminuints les dites gracies, vos poguesets d'aquell 
que le rappelle J. Verdon, ${ }^{31}$ "Un petit traité anonyme écrit vers la fin du XIIIe siècle laisse supposer que les élégantes peuvent passer beaucoup de temps à leur toilette. Il examine en effet successivement l'art de se laver, l'ornement de la chevelure, les cheveux noirs, l'embellissement du visage, la dépilation, la beauté des lèvres, la blancheur des dents, la manière de rendre l'haleine suave, la clarification du teint". Mais cela paraît contredire les préceptes chers aux troubadours, et rejeter la beauté naturelle dans des fantasmes purement littéraires. Cependant, la belle donna est aussi une donna saine, dont la santé est excellente.

Or, les problèmes d'hygiène apparaissent dans les textes littéraires, au détour bien souvent d'une séquence narrative, dans laquelle ils ont alors une fonction particulère, ou pour expliquer l'excellence du portrait de la dame que le troubadour est en train de faire. Dans Jaufre par exemple, les personnages se lavent systématiquement les mains avant de passer à table,

Demandon aiga per lavar,

Qe-s volon metre a manjar. ${ }^{32}$

D'autres mentions identiques scandent ainsi cette histoire. Parfois, les demoiselles s'occupent elle-mêmes de laver leur hôte,

E la donzela es anada

Ves el, que-l servi al lavar. ${ }^{33}$

Souvent, avant le repas, l'auteur précise que les protagonistes présents prennent la peine de se laver, sans préciser d'ailleurs s'il s'agit simplement

servir e ajudar, a sosteniment e secors de vostres persones e gentilesses. [...] E per tal com en lo dit 'Tresor', d'on és la present composició treta, és feta mensió de banys o estubes, e peledors e d'altres cosses per mudificar les inmundicies del cors, e vosaltres ayats e sapiats ya la manera de les cosses, no he curat tractar sinó d'alcuns banys o estubes qui son medicinals per algunes cosses que són nessesaries de saber e ésser fer, e d'alguna singular manera de pelador, e puys de les belessas de la cara e daltres partides del cors, segons que trobaretz per les reseptes presents.

${ }^{31}$ J. VERDON. La femme au Moyen-Age, 1999.

${ }^{32}$ Jaufre, o.c., p. 64.

${ }^{33}$ Jaufre, o.c., p. 272. 
des mains. Cela témoigne cependant d'un rituel habituel compris par le lecteur, car correspondant à un usage quotidien. Il est en effet normal de se laver les mains, ou de donner de l'eau à un hôte avant le repas, geste accompli par la femme. De même, nous l'avons déjà vu, les "caras ben lavadas" permettent à Flamenca et à ses suivantes de ne pas montrer leur désespoir, et sont alors synonymes de gaité, de bien-être, voire de sérénité, ce qu'elles sont loin d'éprouver. Dans ce roman aussi apparaît le rituel du lavage des mains avant le repas.

Au culte de la Vierge, dont l'essor a lieu à partir du XIème siècle, répond celui de la donna, sacralisée par les troubadours, et dont la description peut faire penser à celle de Marie. Son portrait n'est pas réaliste, mais quelques détails, parfois, rappellent que la littérature accomplit une oeuvre de re-création à partir d'un imaginaire qui s'alimente à des sources multiples et variées. Le mythe qui naît alors s'appuie sur la réalité, certes, mais surtout sur des symboles ou des représentations qui ont une valeur édificatrice, puisque cherchant à magnifier un être que la société, par besoin d'asseoir la possession de la terre, et le pouvoir, tente de minoriser. Il n'en reste pas moins que le désir du troubadour, ou ses fantasmes, l'incitent à vouloir contempler la nudité de la femme aimée. Cependant, le vêtement, en suggérant le corps nu, reste un symbole d'appartenance à une classe, et de désir réfréné, et il protège tout en attirant. La description qui se dégage de ces textes, littéraires ou non, renvoie bien une image double, miroir d'un monde où la femme tient une place que lui conteste parfois le système féodal. Mais elle existe aussi, et ses revendications se reflètent bien souvent dans l'apparence qu'elle choisit de montrer, même si, pour cela, et pour correspondre à l'image abstraite et idéalisée de la littérature, elle s'intéresse aux soins esthétiques ou à la médecine afin de trouver des réponses qui lui permettent d'affirmer son identité. 


\section{Résumé}

Le portrait de la donna des troubadours participe des stéréotypes littéraires. Cependant la réalité, différente, renvoie parfois l'image d'une femme qui utilise son apparence pour affirmer son identité. Soins et vêtements rappellent qu'elle a une personnalité que les règles religieuses ou sociales - celles-là même dictées par son statut de femme mariée n'arrivent pas toujours à contenir. Si la littérature offre un champ lexical descriptif somme toute assez restreint, les textes non littéraires ne sont pas vraiment plus prolixes. Cela étant, le portrait littéraire peut aussi puiser dans la diététique, la médecine et les principes d'esthétique afin de rendre à cette femme d'oc un rang que la société cherche à lui confisquer.

\section{Resumo}

O retrato da donna dos trovadores participa dos estereótipos literários. Entretanto, a diferente realidade reflete, por vezes, a imagem de uma mulher que utiliza sua aparência para afirmar sua identidade. Cuidados e roupas lembram que ela tem uma personalidade, que as regras religiosas ou sociais - mesmo sendo ditadas pelo seu status de mulher casada não chegam a fixar. Se, por um lado, a literatura oferece um campo lexical descritivo, bastante restrito, os textos não literários não são, verdadeiramente, mais prolixos. Esse retrato literário pode ser buscado, também, na dietética, na medicina, e nos princípios de estética, na tentativa de devolver à mulher occitana uma posição que a sociedade visa lhe confiscar. 